Check for updates

Cite this: RSC Adv., 2017, 7, 54213

\title{
A novel surface cross-linked GO-based membrane with superior separation performance
}

\begin{abstract}
Zhen Qin, $\dagger$ Lifang Wang, $\uparrow$ Wenzheng Zhang and Kai Pan (iD *
In this study, a novel procedure of fabricating a high performance graphene oxide (GO) composite membrane with high water permeability and selectivity was developed. The GO-based composite membrane, which was fabricated by assembling GO nanosheets on a polyacrylonitrile (PAN) ultrafiltration substrate using vacuum filtration method, was cross-linked by 1,3,5-benzenetricarbonyl trichloride (TMC). Intriguingly, the cross-linking reaction that just occurred at the surface of the GO layer not only provides a stable spacing of the surface GO nanosheets, which ensures the separation accuracy of the composite membrane, but also keeps a high water transport efficiency when passing through the uncross-linked interlamination channels of the GO layer. Pervaporation performance testing, using a water/ethanol feed solution, demonstrated that the separation accuracy and water flux for the optimized surface cross-linked GO composite membrane (TGOm) were significantly better or at least comparable to the values from the GO composite membrane (GOm), and the separation factor of TGOm was enhanced almost several fold. Additionally, with the increasing temperature of the feed solution, the TGOm still maintained an "ideal" separation accuracy, indicating the fine thermostability of the cross-linked membrane.
\end{abstract}

Received 9th October 2017

Accepted 21st November 2017

DOI: $10.1039 / c 7 r a 11088 j$

rsc.li/rsc-advances mechanical strength of the as-prepared GO membrane., Moreover, the hydrophilic functional groups in the GO structure may allow the fast transport of water molecules follow a tortuous pathway but preventing the permeation of larger size and hydrophobic molecules. ${ }^{22-24}$ As confirmed in our previous study, ${ }^{25}$ GO nanosheets can form a unique structure with a hydrophilic and negatively charged "gate" and hydrophobic 2D nanochannels. This unique structure provides a significant improvement for water flux of GO membrane based on the capture and slip flow theory. In these contexts, GO membrane can offer a multitude of advantages in separation applications.

However, when the GO membrane was immersed in a polar solution, the spacing of GO sheets increased due to the hydration of GO, ${ }^{26-30}$ thus makes it a challenge to manipulate the spacing between the GO nanosheets in aqueous solution. Moreover, the GO membrane is likely to partly disperse in water especially under a cross-flow condition that typically encountered in separation applications. ${ }^{25,28}$ As a result, it would be unusually difficult to control the separation accuracy of GO membrane. In actually, two important factors should be considered while GO membrane was used for water separation. One is a nearly frictionless and interconnected permeate channels formed in stacked GO layer, which plays a crucial role for the fast flow of water molecules and facilitates the enhancement of separation efficiency. ${ }^{24,25,31,32}$ The other is that the GO spacing need to be fixed within an appropriately sized range for the purpose of precise sieving, and overcome the hydration force to prevent the membrane dispersion in
State Key Laboratory of Organic-Inorganic Composites, Beijing Key Laboratory of Advanced Functional Polymer Composites, College of Materials Science and Engineering, Beijing University of Chemical Technology, Beijing 100029, China. E-mail: pankai@mail.buct.edu.cn

$\dagger$ The first two authors contributed equally to this work. 
water. $^{33-35}$ At present, many efforts have been performed to control GO spacing and improve the stability of GO membrane by covalently bonding the stacked GO nanosheets with rigid chemical groups, ${ }^{36-41}$ high separation accuracy would be achieved simultaneously. However, it is obviously that the excessive chemical cross-linking of GO nanosheets would cause the detrimental effects on the water permeation of GO membrane. ${ }^{33}$ This contradiction between separation accuracy and water flux is still a challenge need to be addressed for the application of GO membrane in water separation.

In this work, a novel procedure is introduced to fabricate high performance GO composite membrane. First, the GO nanosheets were deposited on a polyacrylonitrile (PAN) ultrafiltration substrate via vacuum filtration approach. And then the as-prepared GO membrane (GOm) was cross-linked by 1,3,5benzenetricarbonyl trichloride (TMC) to obtain the surface cross-linked GO composite membrane (TGOm). It is important that the cross-linking reaction is only occurred at the surface of GO layer, which could solve the contradiction between separation accuracy and permeate flux perfectly. The special structure of this membrane was designed to obtain both high water permeability and selectivity in the application of water separation. The obtained TGOm is characterized and the separation performance is investigated by pervaporation.

\section{Experimental}

\subsection{Materials}

Graphite powder (99.95 wt\%) was provided by Qingdao Henglide graphite Co., Ltd. Sulfuric acid $\left(\mathrm{H}_{2} \mathrm{SO}_{4}, 98 \mathrm{wt} \%\right)$, potassium permanganate $\left(\mathrm{KMnO}_{4},>99.5 \mathrm{wt} \%\right)$, phosphoric acid $\left(\mathrm{H}_{3} \mathrm{PO}_{4},>98 \mathrm{wt} \%\right)$, hydrogen peroxide $\left(\mathrm{H}_{2} \mathrm{O}_{2}, 30 \mathrm{wt} \%\right)$, hydrochloric acid ( $\mathrm{HCl}, 36-38 \mathrm{wt} \%$ ), hexyl hydride (>97 wt\%), ethanol $(\mathrm{EtOH},>99.7 \mathrm{wt} \%)$, sodium hydroxide $(\mathrm{NaOH},>96 \mathrm{wt} \%)$, sodium chloride $(\mathrm{NaCl},>99.5 \mathrm{wt} \%)$ were purchased from Beijing Chemical Co., Ltd. 1,3,5-benzenetricarbonyl trichloride (TMC, 99 wt\%) was supplied by Beijing Bailingwei Technology Co., Ltd. The commercial PAN ultrafiltration membranes (MWCO $=400 \mathrm{kDa}$ ) was obtained from Yizhuoer (Beijing) membrane technology Co., Ltd. All reagents were analytical grade and used as received with no further purification. All of the water used in this work was Milli-Q deionized water (18.1 $\mathrm{M} \Omega \mathrm{cm}$ at $25^{\circ} \mathrm{C}$ ).

\subsection{Preparation of GO}

GO was prepared by exfoliation of graphite following a modified Hummer's method. The mixture of $\mathrm{H}_{2} \mathrm{SO}_{4}(360 \mathrm{~mL})$ and $\mathrm{H}_{3} \mathrm{PO}_{4}$ $(40 \mathrm{~mL})$ was poured into a three-necked flask which was immersed in an ice bath. Graphite ( $3 \mathrm{~g}$ ) was then added into the flask under magnetic stirred. After the addition of $\mathrm{KMnO}_{4}(18 \mathrm{~g})$ tardily, the mixture was stirred continuously at the temperature of $0{ }^{\circ} \mathrm{C}$ for $2 \mathrm{~h}$. Subsequently, the reaction was heated to $35{ }^{\circ} \mathrm{C}$ and stirring was continued for $2 \mathrm{~h}$. Then the temperature was increased to $50{ }^{\circ} \mathrm{C}$ and stirred for additional $12 \mathrm{~h}$. The reactor content was then cooled to room temperature and an amount of ice water $(400 \mathrm{~mL})$ with $30 \% \mathrm{H}_{2} \mathrm{O}_{2}(10 \mathrm{~mL})$ was poured into the resultant mixture sequentially. After the product was washed extensively with hydrochloric acid and deionized water, the resulting solution was collected with centrifugation, ultrasonication and lyophilization to obtain homogeneously dispersed GO aqueous suspensions $\left(500 \mathrm{mg} \mathrm{L}^{-1}\right)$ and GO powder respectively.

\subsection{Fabrication of GO composite membrane (GOm)}

A commercially available porous PAN ultrafiltration membrane was used as the support membrane, and was first immersed into $\mathrm{NaOH}$ solution $\left(1 \mathrm{~mol} \mathrm{~L}^{-1}\right)$ at the temperature of $70{ }^{\circ} \mathrm{C}$ for $20 \mathrm{~min}$ to modify the membrane surfaces by hydrolysis. Then the pretreated PAN ultrafiltration membrane was soaked into deionized water for a certain time to remove residual $\mathrm{NaOH}$. In a typical procedure, $2 \mathrm{~mL}$ of as-prepared GO aqueous suspensions was diluted using deionized water followed by water bath ultrasonication to give a brown GO dispersion. Then the dilute GO aqueous solution was vacuum filtrated onto the surface of the modified PAN ultrafiltration membrane support until it was completely waterless to depositing a GO multilayer.

\subsection{Synthesis of the surface cross-linked GO composite membrane (TGOm)}

In order to avoid the degeneration of TMC in water as the existence of acyl chloride group, TMC powder (0.1 g) was entirely dissolved in hexyl hydride $(40 \mathrm{~mL})$ at room temperature through magnetic stirred. The as-prepared GOm was fixed in a vacuum filtration unit, then the aforesaid TMC/hexyl hydride mixtures were poured into the filtering cup, the cross-linking reaction was continued at normal temperature for $3 \mathrm{~min}$. Subsequently, the obtained TGOm was taken out and left to dry naturally. In addition, to investigate the effect of the dosage of TMC used during the cross-linking reaction on the separation performance of the membrane, different proportions of TMC amount to GO (i.e., $100: 1,80: 1,60: 1,50: 1,25: 1$ ) was designed to synthesize TGOm for further pervaporation experiments.

\subsection{Evaluation for the separation performance of GOm and TGOm}

A laboratory scale pervaporation unit was used for the pervaporation separation testing of GOm and TGOm. In pervaporation, the membrane with an effective surface area of $14.7 \mathrm{~cm}^{2}$ was mounted in the middle of a pervaporation cell. The tests were conducted with the following feed solution in the listed order, one at a time: water/ethanol mixtures with varied proportion $(30 / 70,97 / 3)$. During the experimental process, the feed solution was preheated to $30{ }^{\circ} \mathrm{C}$ in a water bath and pumped to the pervaporation cell using a Langer® peristaltic pump. The permeate side of the membrane cell was connected to a vacuum pump, which provided a driving force made the pressure maintained under $100 \mathrm{~Pa}$ during the permeation separation. The pervaporation separation process was performed following three steps based on the solution-diffusion model: the feed solution in direct contacts with the upstream face of the membrane and dissolves onto the membrane 
surface; then diffuses across the membrane as a pressure gradient exists; and finally evaporates at the downstream side of the membrane. The vapour stream passes through a condenser surrounded by liquid nitrogen and the condensate is collected for a period of time to obtain permeate sample for concentration analysis.

The pure water flux $(J)$ was calculated from the following equation:

$$
J=\frac{M}{A t}
$$

where $M$ is the mass of the permeate collected in the cold trap, $A$ is the effective membrane area and $t$ is the experimental time.

The separation efficiency of the membrane was determined by the weight fraction of water in the permeation. With gas chromatography (GC-9900), the permeate compositions were determined.

The pervaporation separation factor (SF) of the mixture contained ingredient $\mathrm{A}$ and $\mathrm{B}\left(\alpha_{\mathrm{AB}}\right)$ was obtained from the following equation:

$$
\alpha_{\mathrm{AB}}=\frac{Y_{\mathrm{A}} / Y_{\mathrm{B}}}{X_{\mathrm{A}} / X_{\mathrm{B}}}
$$

where $Y_{\mathrm{A}}$ and $Y_{\mathrm{B}}$ are the mole fractions of $\mathrm{A}$ and $\mathrm{B}$ in the feed solution respectively, $X_{\mathrm{A}}$ and $X_{\mathrm{B}}$ are the mole fractions of A and $\mathrm{B}$ in the permeate.

Generally, the ingredient which has a higher permeation rate was denoted as $\mathrm{A}$, therefore the quantitative value of $\alpha_{\mathrm{AB}}$ is greater than 1.

To evaluate the thermostability of the TGOm during the pervaporation experiment, feed solutions with different temperature range from 30 to $60{ }^{\circ} \mathrm{C}$ was performed, and testing once at intervals of $10^{\circ} \mathrm{C}$.

\section{Results and discussion}

\subsection{Characterization of GO structure}

The physicochemical properties of GO samples prepared using a modified Hummer's method were characterize with several different techniques. Fig. 1a shows the scanning electron microscope (SEM) images of the exfoliated GO thin flakes, which displays a typical wave winkles and a large lateral size about $100 \mu \mathrm{m}$. Such a large lateral size of GO will facilitate the fabrication of a GO membrane by vacuum filtration to form the layered structure. ${ }^{25}$

As shown in Fig. 1b, the Fourier transform infrared (FT-IR) spectrum of GO represents strong bands centered at $3399 \mathrm{~cm}^{-1}$ corresponds to hydroxyl $(\mathrm{O}-\mathrm{H})$ stretching vibration. The peaks at $1730 \mathrm{~cm}^{-1}$ is assigned to carbonyl $(\mathrm{C}=\mathrm{O})$ absorption and $1622 \mathrm{~cm}^{-1}$ is related to the unoxidized $\mathrm{sp}^{2} \mathrm{C}=\mathrm{C}$ bonds in the carbon lattice. ${ }^{42}$ The peaks at 1383, 1227 and $1071 \mathrm{~cm}^{-1}$ can be attributed to the carboxy $\mathrm{C}-\mathrm{O}$ stretching vibration, $\mathrm{C}-\mathrm{OH}$ stretching vibration, epoxy $\mathrm{C}-\mathrm{O}-\mathrm{C}$ stretching vibration, respectively. ${ }^{\mathbf{4 3 4} 4}$ The FT-IR results demonstrated the presence of oxygen-containing functional groups on the GO sheets, which may increase the hydrophilic and enable the dispersion of GO in water. ${ }^{45}$
Fig. 1c gives the X-ray diffraction (XRD) patterns of GO nanosheets, a diffraction peak at $2 \theta=10.7^{\circ}$ can be observed, corresponding to the (001) peak of graphitic carbon plane, indicates an interlayer spacing of about 8.29 $\AA$. The large $d$ spacing of GO sheets were attributed to the oxygenated functional groups introduced in GO during the chemical oxidation..$^{6,7,46}$

The X-ray photoelectron spectra (XPS) were used to further characterize the chemical bonds in GO. Four types of $\mathrm{C}$ bonds were distinguished from the curve fitting of $\mathrm{C}$ 1s spectra (Fig. 1d), the binding energy of $284.7 \mathrm{eV}$ is assigned to $\mathrm{C}-\mathrm{C} / \mathrm{C}=$ $\mathrm{C}$, and $286.5 \mathrm{eV}$ is ascribed to $\mathrm{C}-\mathrm{O}$. The respective binding energy of 287.6 and $288.8 \mathrm{eV}$ correspond to $\mathrm{C}=\mathrm{O}$ and $\mathrm{COOH}^{47}$ The $\mathrm{C} / \mathrm{O}$ atomic ratio determined for the GO sheets is 2.03. Peak intensities of intact $\mathrm{C}$ and oxygenated $\mathrm{C}$ atoms in the XPS spectrum were 34.76 and $65.24 \%$, respectively. The above XPS results mean that substantially oxidized GO had been prepared following the modified Hummer's method. ${ }^{48}$

\subsection{Preparation of GOm and TGOm}

A porous supporting layer and a functional barrier layer constitute the two principal part of the composite separation membrane. ${ }^{49}$ In our experiments, a commercial polyacrylonitrile (PAN) ultrafiltration substrate was employed as the support for GO barrier layer. To ensure membrane stability, for the first step, PAN substrates were hydrolyzed by $\mathrm{NaOH}$ solution to create functional active sites $\left(-\mathrm{COOH}\right.$ or $\left.\mathrm{CONH}_{2}\right)$ on the supporting membrane, so that the GO layers could be strongly adhere to PAN substrates through hydrogen bonds. ${ }^{50,51}$ After vacuum filtrate and drying in a vacuum, the GO composite membrane (GOm) with excellent flexibility and stability was obtained, as shown in Fig. 2. The inter-sheet $d$-spacing in the barrier layer of the GOm can be adjusted by modifying the spacing with chemicals. Here, TMC was chose as cross-linker to immobilize the adjacent GO nanosheets and insure the stability of GO membrane in water. The top surface of GOm was soaked in the TMC/hexyl hydride mixtures, then the cross-linking reaction occurred subsequently. We noticed that 3 minutes vacuum filtration process can't make the TMC/hexyl hydride mixtures infiltrate into the interlamination channels of deeper GO layers, thus only several GO nanosheets of the surface GO layers were cross-linked by chemical bonds. The enlarged view in Fig. 2 gives the micro-structure model of GO membrane, in which the acyl chloride groups in TMC reacted with the hydroxyl groups on GO planes as well as the carboxyl groups at the edges to form ester and anhydride bonds. The reaction mechanism further revealed that the GO nanosheets at the edge of the integral TGOm was also cross-linked, which provided a stable spacing of the GO nanosheets thus restrained the swelling of TGOm in water, while hold the interlamination channels exist in the internal of GO layer.

The FT-IR spectra was used to characterize the functional groups of the top and bottom surface in TGOm. As shown in Fig. 3a, the bottom surface of TGOm (B-TGOm) lead to very typical FT-IR spectra that consistent with GO. However, the FTIR spectra of the top surface of TGOm (T-TGOm) reveal 

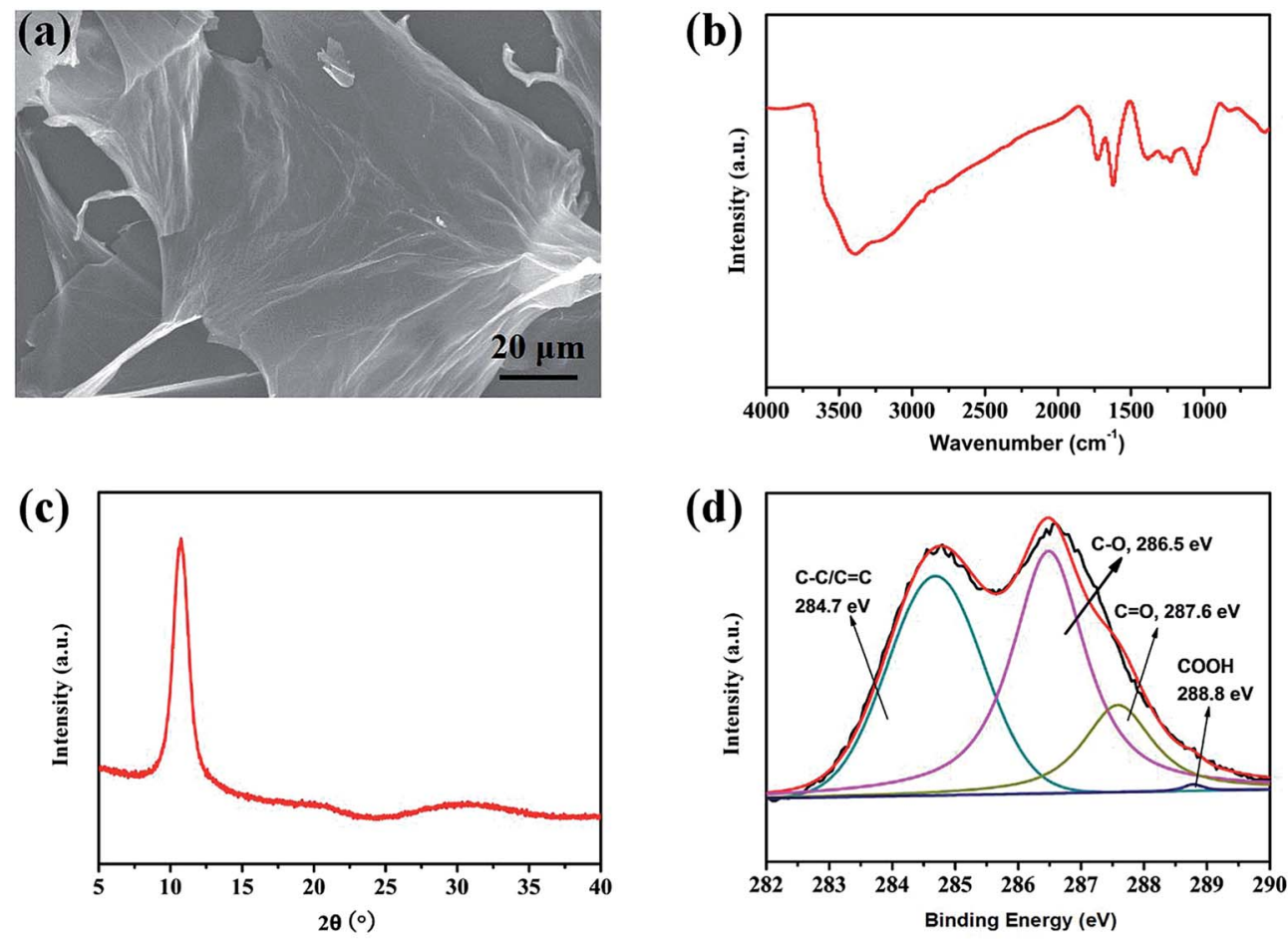

Fig. 1 (a) SEM, (b) FT-IR spectra, (c) XRD pattern, and (d) XPS elemental analyses in the C 1s region of GO nanosheets.

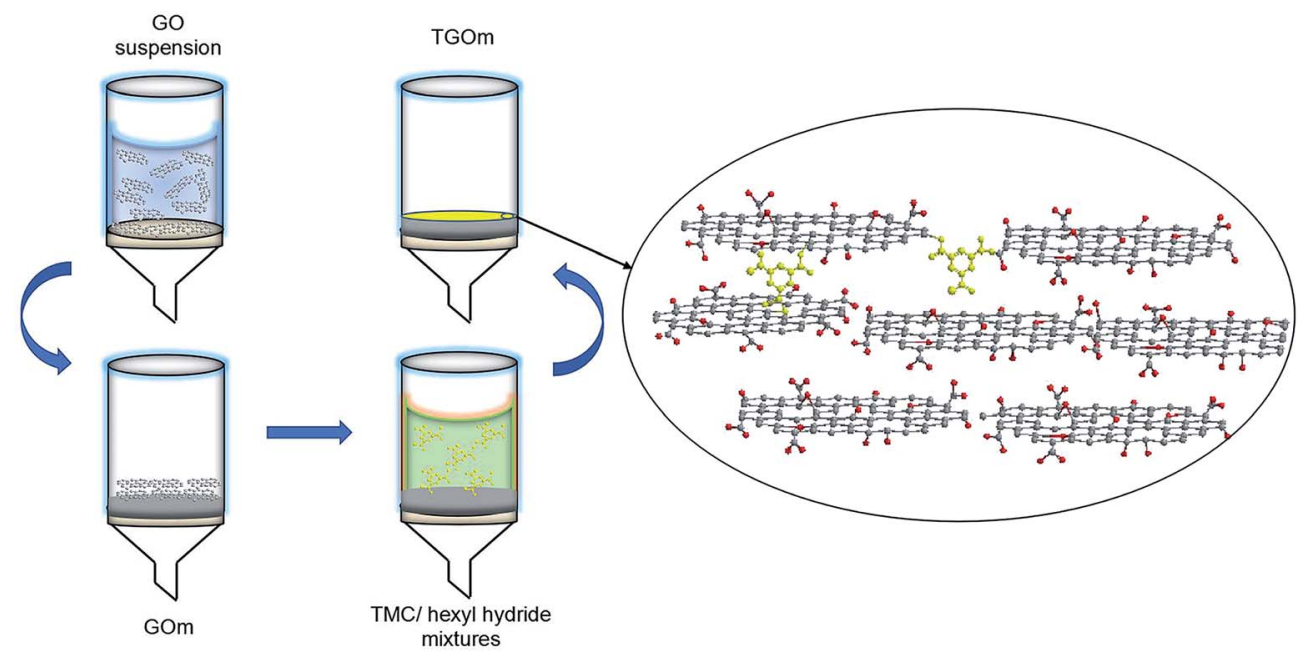

Fig. 2 Schematic illustration of the preparation process of GOm and TGOm, and the mechanism of reactions between GO and TMC.

a differentiating peak at $1795-1830 \mathrm{~cm}^{-1}$, which is absent from the spectra of the bottom surface of TGOm. This peak represents the stretching vibration of $\mathrm{C}=\mathrm{O}$ bands in ester and anhydrides groups, which could result from the reactions between TMC and GO. The $\mathrm{C}=\mathrm{O}$ bands were also present in carboxyl groups of original GO and unreacted acyl chloride groups in TMC. The XPS characterization show the elementary compositions of the GOm and TGOm. It is observed in Fig. $3 \mathrm{~b}$ that the $\mathrm{C}$ and $\mathrm{O}$ atomic percentages of TGOm is higher than that of GOm, this could attribute to the addition of TMC within TGOm. A small quantity of the C 1 elementary that arise in
TGOm were possibly derived from unreacted acyl chloride groups in TMC, and this results show a close agreement with the analysis of FT-IR. The XPS were used to further characterize the chemical bonds in TGOm. Four types of $\mathrm{C}$ bonds were distinguished from the curve fitting of $\mathrm{C}$ 1s spectra (Fig. 3c), the binding energy of $284.5 \mathrm{eV}$ is assigned to $\mathrm{C}-\mathrm{C} / \mathrm{C}=\mathrm{C}$, and $286.6 \mathrm{eV}$ is ascribed to $\mathrm{C}-\mathrm{O}$. The respective binding energy of 288.1 and $288.8 \mathrm{eV}$ correspond to $\mathrm{C}=\mathrm{O}$ and COOR, and the binding energy of COOR is higher than that of GO. The above XPS results mean that GOm had cross-linking by TMC. The XRD patterns of the GOm and TGOm are compared in Fig. 3d. The 
(a)

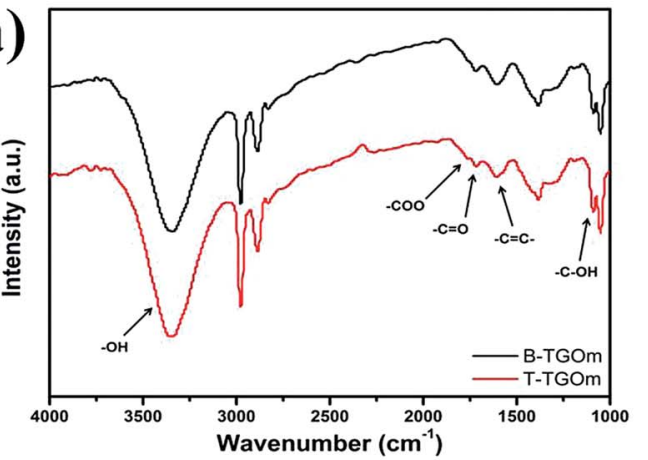

(c)

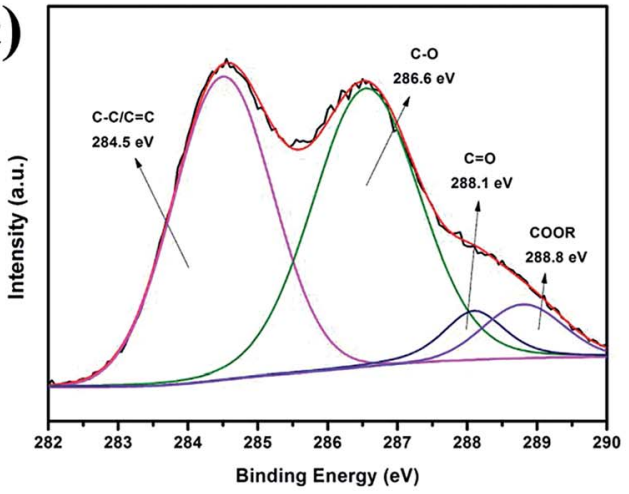

(b)

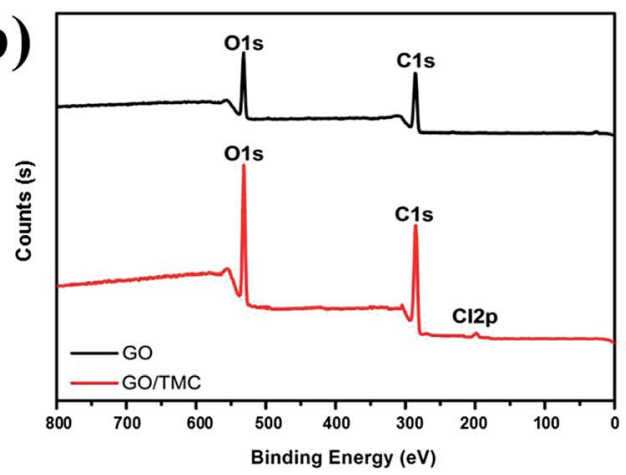

(d)

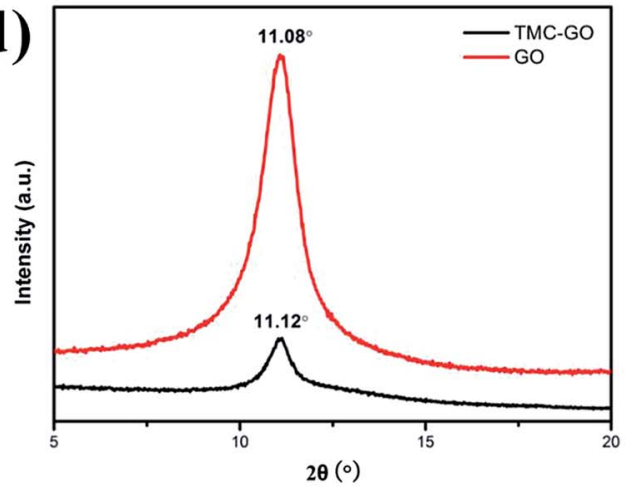

Fig. 3 (a) FT-IR spectra, (b) XPS survey of GOm and TGOm, (c) XPS elemental analyses in the C 1s region of TGOm, (d) XRD pattern.

GOm and TGOm have a $d$-spacing value of $7.98 \AA$ and $7.95 \AA$, respectively. This results show the TMC isn't infiltrate into the interlamination channels of deeper GO layers, there is only surface reaction between GOm and TMC.

\subsection{Morphology and microstructure of GOm and TGOm}

The surface and cross-sectional morphologies of the GOm and TGOm were examined by the SEM, as depicted in Fig. 4. From the surface images of GOm (Fig. 4a) and TGOm (Fig. 4c), we can see very similar morphologies which displays a typical wrinkled GO skin layers. Fig. $4 \mathrm{~d}$ and e show the cross-section of GOm and TGOm, respectively. The stacked lamellar structure at the upper layer and macroporous voids at the substratum can be observed clearly, indicating the discrete GO layer supported by PAN ultrafiltration substrate, which is similar to our previous studies. ${ }^{50}$ It did not see any significant changes in the thickness of GO layer after TMC-cross-linking reaction. The surface of TGOm was relatively hydrophobic with a water contact angle of $57^{\circ}$ (Fig. $4 \mathrm{c}$ inset), which was higher than that of GOm about $51^{\circ}$ (Fig. 4a inset). The decrease of membrane hydrophilicity may due to the TMC used as a cross-linker.

\subsection{Pervaporation dehydration performance}

Membranes that could reach superior separation performance should be provided with high permeation flux and separation accuracy simultaneously. To this end, the design and tailoring of membrane permeation channels need to be taken seriously, as it is crucial for precise separation of ions or molecules as well as high-efficiency of water transport. ${ }^{52}$ Here, we designed the surface cross-linked GO barrier layer for precise separation which remains the uncross-linked interlamination channels for water transportation. The effect of the dosage of TMC used during the cross-linking reaction on the separation performance of the GO-based membrane was investigated, using 70/ 30 of water/ethanol as feed solution at $30^{\circ} \mathrm{C}$.

Fig. 5a shows the permeate flux and water concentration in permeate results for the GOm and the TGOm with different mass ratio of TMC to GO. The date revealed that the water concentration in permeate increased markedly from $87.9 \%$ (for GOm) to $97.3 \%$ (for TGOm) when the mass ratio of TMC to GO is $60: 1$. However, the water flux of TGOm changed slightly into $0.99 \mathrm{~L} \mathrm{~m}^{-2} \mathrm{~h}^{-1}$, which was at least comparable to the value of GOm as $1.19 \mathrm{~L} \mathrm{~m}^{-2} \mathrm{~h}^{-1}$. It was found that, with the increasing of the mass ratio of TMC to GO, the water concentration in permeate maintained at a high level, while the permeate flux varied rather minimal. As can be seen that the water concentration in permeate and the permeate flux of the tested TGOm reached $97.2 \%$ and $1.06 \mathrm{~L} \mathrm{~m}^{-2} \mathrm{~h}^{-1}$, respectively, when the mass ratio of TMC to GO reached to $100: 1$. In addition, the calculative SF results obtained from the eqn (2) indicated that the TGOm had a SF value of 81 which much higher than that for GOm of 17. The above results demonstrated that the TGOm exhibited a better separation performance than the GOm.

The separation performance of TGOm with the TMC/GO mass ratio of $100: 1$ was also examined using feed solution of $97 / 3$ of water/ethanol. As shown in Fig. 5b, the water concentration in permeate of TGOm has reached $99.5 \%$, better than the result of $99 \%$ for GOm. The water flux of the tested TGOm was slightly down to $5.9 \mathrm{~L} \mathrm{~m}^{-2} \mathrm{~h}^{-1}$ when compared with the 

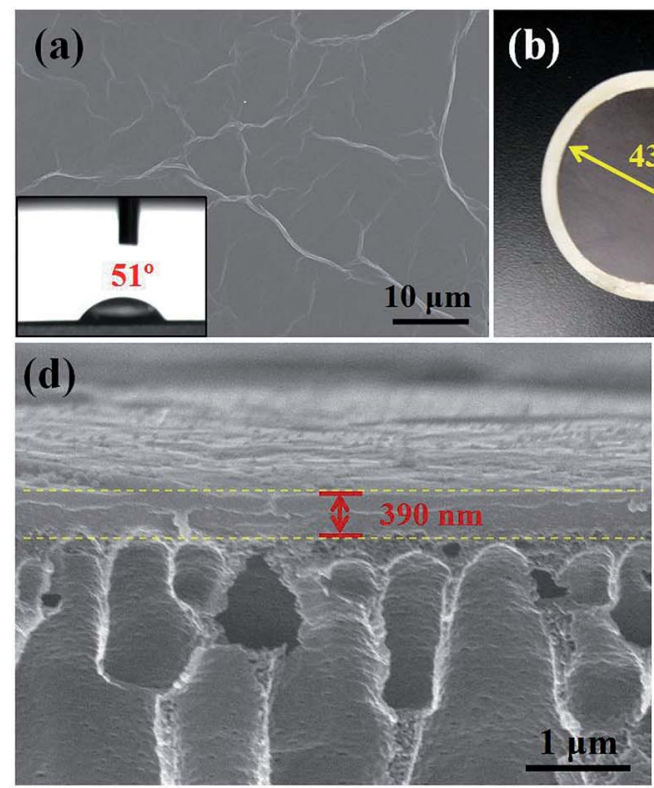
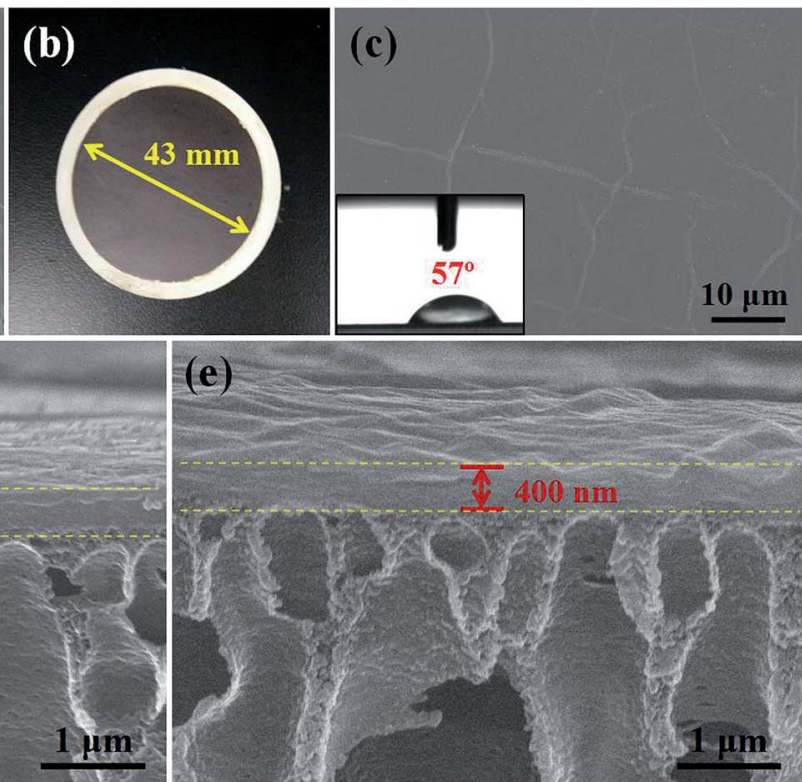

Fig. 4 (a) Surface and (d) cross-section SEM images of the GOm; (c) surface and (e) cross-section SEM images of the TGOm (inset: water contact angle); (b) the digital photographs of TMC-cross-linked GO/PAN composite membrane (TGOm).
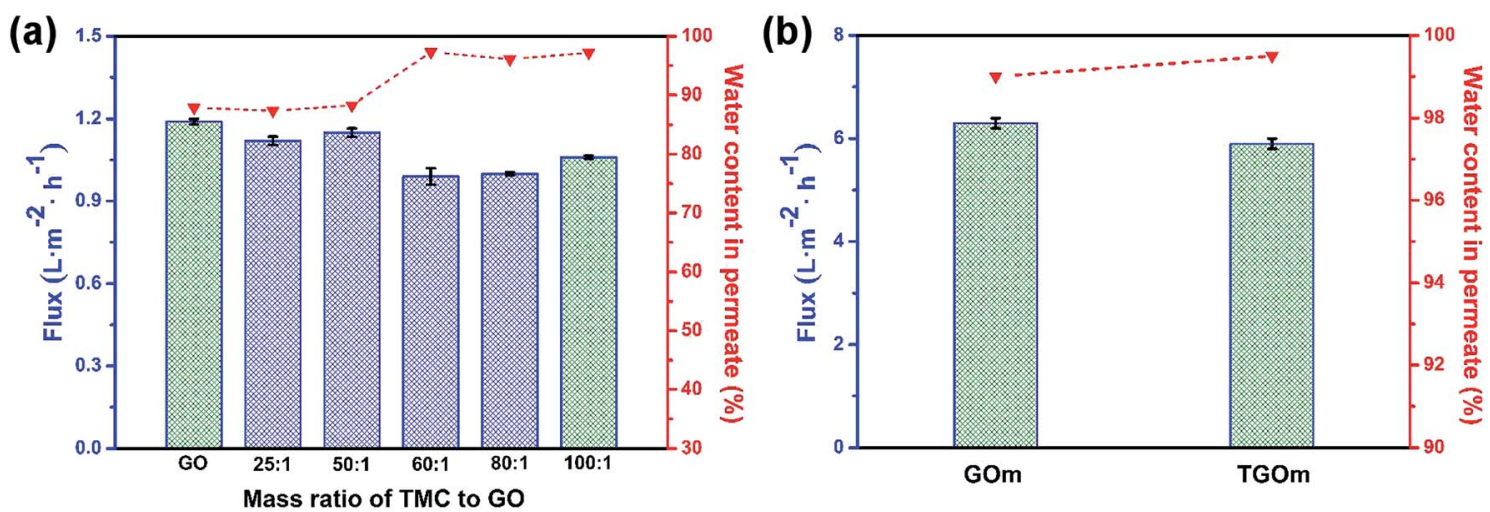

Fig. 5 The pervaporation performance for: (a) GOm and TGOm with different mass ratio of TMC to GO using 70/30 of water/ethanol as feed solution; (b) GOm and TGOm with the mass ratio of TMC to GO at 100:1 using 97/3 of water/ethanol feed solution.

result of $6.3 \mathrm{~L} \mathrm{~m}^{-2} \mathrm{~h}^{-1}$ for GOm. It could be attributed to the cross-linking of GO nanosheets within the TGOm which result in the smaller nanochannel for water transportation. Nevertheless, the calculated SF value of 6.2 for TGOm was twice higher than 3.1 from the data of GOm, demonstrated that the TGOm was more suitable for the ethanol dehydrate application.

In general, for composite separating membranes, the permeation flux and selectivity for molecules are the primary criterions that determined their separation performance, and it shows negative correlation between the two criterions. ${ }^{53}$ However, the experimental data of the tested TGOm in our investigation verified that the TGOm could reach superior separation performance both with high water flux and selectivity for ethanol. This observation could be attributed to the special structure as we designed for TGOm with a surface cross-linked GO barrier layer and the uncross-linked interlamination channels.
As we know, the performance of GO-based membrane at high temperature could become unstable due to the slight decomposition of the GO. In our experiment, the separation performance of the TGOm was examined using 97/3 of water/ ethanol as feed solution under different temperature to better understand the stability of TGOm for ethanol dehydration. The permeate flux and the water concentration in permeate results for TGOm are shown in Fig. 6 . The data revealed that the TGOm had an increasing water flux from 6.3 to $14 \mathrm{~L} \mathrm{~m}^{-2} \mathrm{~h}^{-1}$ as the operating temperature raised from 30 to $60^{\circ} \mathrm{C}$. One explanation for this observation is that the viscosity of the feed solution decreased under higher temperature result in the enhanced diffusion coefficient, thus the water permeation accelerated along with the rise of the operating temperature and the permeate flux increased. Whereas the operating temperature raised constantly, the water concentration in permeate values of 


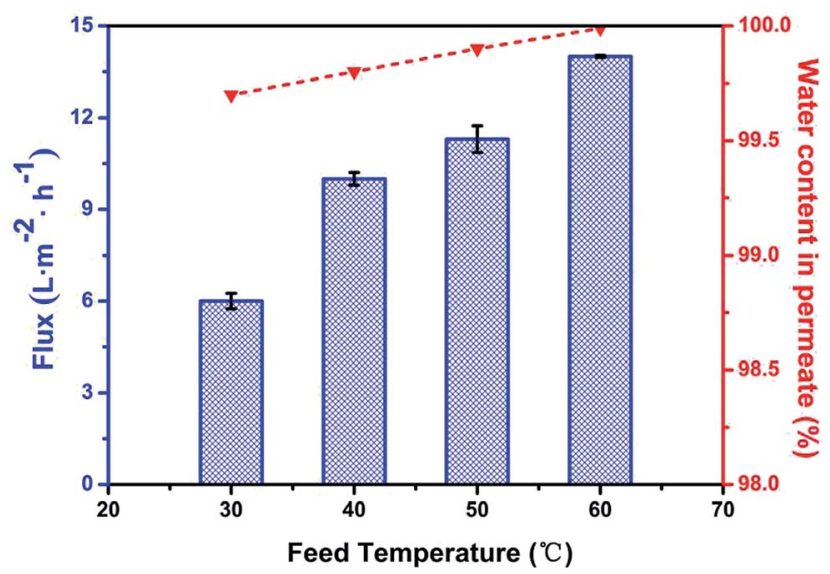

Fig. 6 The pervaporation performance of the TGOm at different feed temperature using $97 / 3$ of water/ethanol feed solution.

the tested TGOm always keep up to $99.7 \%$ (at $30{ }^{\circ} \mathrm{C}$ ) until reached the detection limited value of $99.99 \%$ (at $60{ }^{\circ} \mathrm{C}$ ). It turned out that the performance of the TGOm at higher temperature had not been affected for the separation of ethanol/water solution effectively. We verified that the crosslinking modification on GO did significant contribute toward the mechanical property and stability during pervaporation.

\section{Conclusions}

In summary, a novel surface TMC-cross-linked GO/PAN composite membrane has been successfully fabricated by vacuum suction method. The surface cross-linked GO barrier layer of TGOm with a stable interlamellar spacing was formed ensuring the precise separation, while it remains the uncrosslinked interlamination channels for the fast water transportation. The pervaporation results indicated that the TGOm exhibits superior separation performance. With the TMC/GO mass ratio increased to $100: 1$, the water concentration in permeate of the TGOm reached at a high level of $97.3 \%$, while the water flux changed slightly to $1.06 \mathrm{~L} \mathrm{~m}^{-2} \mathrm{~h}^{-1}$. The separation factor of 81 for TGOm was nearly five times higher than that of the GOm. Due to the cross-linking of GO nanosheets that prevented the decomposition of the GO layer, the performance of TGOm under different operating temperature from 30 to $60{ }^{\circ} \mathrm{C}$ was stable with a highest water concentration in permeate of $99.7 \%$. This work provides a novel strategy for preparing surface cross-linked GO-based composite membrane with both high water permeability and selectivity for ethanol, as well as fine thermo-stability. The superior performance of this membrane promises it great potential for water separation applications.

\section{Conflicts of interest}

There are no conflicts to declare.

\section{Acknowledgements}

This work was financially supported by the opening foundation of State Key Laboratory of Organic-Inorganic Composites (201701012).

\section{References}

1 D. R. Dreyer, S. Park, C. W. Bielawski and R. S. Ruoff, The chemistry of graphene oxide, Chem. Soc. Rev., 2010, 39, 228-240.

2 D. Li and R. B. Kaner, Graphene-based materials, Science, 2008, 320, 1170-1171.

3 J. Kim, L. J. Cote and J. X. Huang, Two dimensional soft material: new faces of graphene oxide, Acc. Chem. Res., 2012, 45, 1356-1364.

4 W. S. Hummers and R. E. Offeman, Preparation of graphitic oxide, J. Am. Chem. Soc., 1958, 80, 1339.

5 D. C. Marcano, D. V. Kosynkin, J. M. Berlin, A. Sinitskii, Z. Z. Sun, A. Slesarev, L. B. Alemany, W. Lu and J. M. Tour, Improved synthesis of graphene oxide, ACS Nano, 2010, 4, 4806-4814.

6 Y. Zhu, S. Murali, W. Cai, X. Li, J. W. Suk, J. R. Potts and R. S. Ruof, Graphene and graphene oxide: synthesis, properties, and applications, Adv. Mater., 2010, 22, 1-19.

7 K. P. Loh, Q. Bao, G. Eda and M. Chhowalla, Graphene oxide as a chemically tunable platform for optical applications, Nat. Chem., 2010, 2, 1015-1024.

8 F. Kim, L. J. Cote and J. Huang, Graphene oxide: surface activity and two-dimensional assembly, Adv. Mater., 2010, 22, 1954-1958.

9 D. A. Dikin, S. Stankovich, E. Zimney, R. D. Piner, G. H. B. Dommett, G. Evmenenko, S. Nguyen and R. Ruoff, Preparation and characterization of graphene oxide paper, Nature, 2007, 448, 457-460.

10 G. Eda and M. Chhowalla, Chemically derived graphene oxide: towards large-area thin-film electronics and optoelectronics, Adv. Mater., 2010, 22, 2392-2415.

11 Y. Zhu, D. K. James and J. M. Tour, New routes to graphene, graphene oxide and their related applications, Adv. Mater., 2012, 24, 4924-4955.

12 Y. Zhang, S. Zhang and T. S. Chung, Nanometric Graphene Oxide Framework Membranes with Enhanced Heavy Metal Removal via Nanofiltration, Environ. Sci. Technol., 2015, 49, 10235-10242.

13 A. K. Mishra and S. Ramaprabhu, Functionalized graphene sheets for arsenic removal and desalination of sea water, Desalination, 2011, 282, 39-45.

14 K. Goh, L. Setiawan, L. Wei, R. Si, A. G. Fane, R. Wang and Y. Chen, Graphene oxide as effective selective barriers on a hollow fiber membrane for water treatment process, $J$. Membr. Sci., 2015, 474, 244-253.

15 C. M. Chen, Q. H. Yang, Y. G. Yang, W. Lv, Y. F. Wen, P. X. Hou, M. Z. Wang and H. M. Cheng, Self-assembled free-standing graphite oxide membrane, Adv. Mater., 2009, 21, 3007-3011. 
16 H. Li, Z. N. Song, X. J. Zhang, Y. Huang, S. G. Li, Y. T. Mao, H. J. Ploehn, Y. Bao and M. Yu, Ultrathin, molecular-sieving graphene oxide membranes for selective hydrogen separation, Science, 2013, 342, 95-98.

$17 \mathrm{M}$. Hu and B. Mi, Layer-by-layer assembly of graphene oxide membranes via electrostatic interaction, J. Membr. Sci., 2014, 469, 80-87.

18 B. X. Mi, Graphene Oxide Membranes for Ionic and Molecular Sieving, Science, 2014, 343, 740-742.

19 S. P. Koenig, L. D. Wang, J. Pellegrino and J. S. Bunch, Selective molecular sieving through porous graphene, Nat. Nanotechnol., 2011, 7, 728-732.

20 R. R. Nair, H. A. Wu, P. N. Jayaram, I. V. Grigorieva and A. K. Geim, Unimpeded permeation of water through helium-leak-tight graphene-based membranes, Science, 2012, 335, 442-444.

21 H. Chen, M. B. Muller, K. J. Gilmore, G. Wallace and D. Li, Mechanically strong, electrically conductive, and biocompatible graphene paper, Adv. Mater., 2008, 20, 3557-3561.

22 W. D. Boukhvalov, M. I. Katsnelson and Y.-W. Son, Origin of anomalous water permeation through graphene oxide membrane, Nano Lett., 2013, 13, 3930-3935.

23 R. K. Joshi, P. Carbone, F. C. Wang, V. G. Kravets, Y. Su, I. V. Grigorieva, H. A. Wu, A. K. Geim and R. R. Nair, Precise and ultrafast molecular sieving through graphene oxide membranes, Science, 2014, 343, 752-754.

24 B. Liang, P. Zhang, J. Q. Wang, J. Qu, L. F. Wang, X. X. Wang, C. F. Guan and K. Pan, Membranes with selective laminar nanochannels of modified reduced graphene oxide for water purification, Carbon, 2016, 103, 94-100.

25 J. Q. Wang, P. Zhang, B. Liang, Y. X. Liu, T. Xu, L. F. Wang, B. Cao and K. Pan, Graphene oxide as an effective barrier on a porous nanofibrous membrane for water treatment, ACS Appl. Mater. Interfaces, 2016, 8, 6211-6218.

26 T. Szabo, O. Berkesi, P. Forgo, K. Josepovits, Y. Sanakis, D. Petridis and I. Dekany, Evolution of surface functional groups in a series of progressively oxidized graphite oxides, Chem. Mater., 2006, 18, 2740-2749.

27 O. C. Compton and S. T. Nguyer, Graphene oxide, highly reduced graphene oxide, and graphene: versatile building blocks for carbon-based materials, Small, 2010, 6, 711-723.

28 A. Klechikov, J. Yu, D. Thomas and A. V. Talyzin, Structure of graphene oxide membranes in solvents and solutions, Nanoscale, 2015, 7, 15374-15384.

29 A. V. Talyzin, A. Klechikov, M. Korobov, A. T. Rebrikova, N. V. Avramenko, M. F. Gholami, N. Severin and J. P. Rabe, Delamination of graphite oxide in a liquid upon cooling, Nanoscale, 2015, 7, 12625-12630.

30 A. V. Talyzin, S. M. Luzan, T. Szabo, D. Chernyshev and V. Dmitriev, Temperature dependent structural breathing of hydrated graphite oxide in $\mathrm{H}_{2} \mathrm{O}$, Carbon, 2011, 49, 18941899.

31 P. Sun, H. Liu, K. Wang, M. Zhong, D. Wu and H. Zhu, Selective ion transport through functionalized graphene membranes based on delicate ion-graphene interactions, $J$. Phys. Chem. C, 2014, 118, 19396-19401.
32 Z. Wang, R. Sahadevan, C. N. Yeh, T. J. Menkhaus, J. X. Huang and H. Fong, Hot-pressed polymer nanofiber supported graphene membrane for high-performance nanofiltration, Nanotechnology, 2017, 28, 31LT02.

33 M. L. Bruening and D. M. Sullivan, Enhancing the iontransport selectivity of multilayer polyelectrolyte membranes, Chem.-Eur. J., 2002, 8, 3833-3837.

34 E. N. Wang and R. Karnik, Water desalination: Graphene cleans up water, Nat. Nanotechnol., 2012, 7, 552-554.

35 W. S. Hung, C. H. Tsou, M. D. Guzman, Q. F. An, Y. L. Liu, Y. M. Zhang, C. C. Hu, K. R. Lee and J. Y. Lai, Crosslinking with diamine monomers to prepare composite graphene oxide-framework membranes with varying $d$ spacing, Chem. Mater., 2014, 26, 2983-2990.

36 S. Stankovich, D. A. Dikin, O. C. Compton, G. H. B. Dommett, R. S. Ruoff and S. B. T. Nguyen, Systematic post-assembly modification of graphene oxide paper with primary alkylamines, Chem. Mater., 2010, 22, 4153-4157.

37 S. Park, K. S. Lee, G. Bozoklu, W. Cai, S. T. Nguyen and R. S. Ruoff, Graphene oxide papers modified by divalent ions-enhancing mechanical properties via chemical crosslinking, ACS Nano, 2008, 2, 572-578.

38 Y. Tian, Y. Cao, Y. Wang, W. Yang and J. Feng, Realizing ultrahigh modulus and high strength of macroscopic graphene oxide papers through crosslinking of musselinspired polymers, Adv. Mater., 2013, 25, 2980-2983.

39 S. Park, D. A. Dikin, S. T. Nguyen and R. S. Ruoff, Graphene oxide sheets chemically cross-linked by polyallylamine, $J$. Phys. Chem. C, 2009, 113, 15801-15804.

40 C. Cheng, L. D. Shen, X. F. Yu, Y. Yang, X. Li and X. F. Wang, Robust construction of a graphene oxide barrier layer on a nanofibrous substrate assisted by the flexible poly(vinylalcohol) for efficient pervaporation desalination, J. Mater. Chem. A, 2017, 5, 3558-3568.

41 D. Hua, R. K. Rai, Y. Zhang and T. S. Chung, Aldehyde functionalized graphene oxide frameworks as robust membrane materials for pervaporative alcohol dehydration, Chem. Eng. Sci., 2017, 161, 341-349.

42 D. W. Lee, V. L. De Los Santos, J. W. Seo, L. L. Felix, D. A. Bustamante, J. M. Cole and C. H. Barnes, The structure of graphite oxide: investigation of its surface chemical groups, J. Phys. Chem. B, 2010, 114, 5723-5728.

43 Y. Gao, L. Q. Liu, S. Z. Zu, K. Peng, D. Zhou, B. H. Han and Z. Zhang, The effect of interlayer adhesion on the mechanical behaviors of macroscopic graphene oxide papers, ACS Nano, 2011, 5, 2134-2141.

$44 \mathrm{Y}$. Si and E. T. Samulski, Synthesis of Water Soluble Graphene, Nano Lett., 2008, 8, 1679-1682.

45 T. M. Yeh, Z. Wang, D. Mahajan, B. S. Hsiao and B. Chu, High flux ethanol dehydration using nanofibrous membranes containing graphene oxide barrier layers, $J$. Mater. Chem. A, 2013, 1, 12998-13003.

46 J. Chen, B. Yao, C. Li and G. Shi, An improved Hummers method for eco-friendly synthesis of graphene oxide, Carbon, 2013, 64, 225-229. 
47 L. Chen, Z. Xu, J. Li, Y. Li, M. Shan, C. Wang, Z. Wang, Q. Guo, L. Liu, G. Chen and X. Qian, A facile strategy to prepare functionalized graphene via intercalation, grafting and self-exfoliation of graphite oxide, J. Mater. Chem., 2012, 22, 13460-13463.

48 T. Xu, Q. Meng, T. Shen and B. Cao, Facile synthesis of submillimeter sized graphene oxide by a novel method, RSC Adv., 2015, 5, 73737-73741.

49 K. Huang, G. P. Liu, Y. Y. Lou, Z. Y. Dong, J. Shen and W. Q. Jin, A graphene oxide membrane with highly selective molecular separation of aqueous organic solution, Angew. Chem., Int. Ed., 2014, 53, 6929-6932.

50 B. Liang, W. Zhan, G. Qi, S. Lin, Q. Nan, Y. Liu, B. Cao and K. Pan, High performance graphene oxide/polyacrylonitrile composite pervaporation membranes for desalination applications, J. Mater. Chem. A, 2015, 3, 5140-5147.

51 W. S. Hung, C. H. Tsou, M. D. Guzman, Q. F. An, Y. L. Liu, Y. M. Zhang, C. C. Hu, K. R. Lee and J. Y. Lai, CrossLinking with Diamine Monomers To Prepare Composite Graphene Oxide-Framework Membranes with Varying $d$ Spacing, Chem. Mater., 2014, 26, 2983-2990.

52 Z. Q. Jia and W. X. Shi, Tailoring permeation channels of graphene oxide membranes for precise ion separation, Carbon, 2016, 101, 290-295.

$53 \mathrm{M}$. $\mathrm{Hu}$ and B. X. Mi, Layer-by-layer assembly of graphene oxide membranes via electrostatic interaction, J. Membr. Sci., 2014, 469, 80-87. 